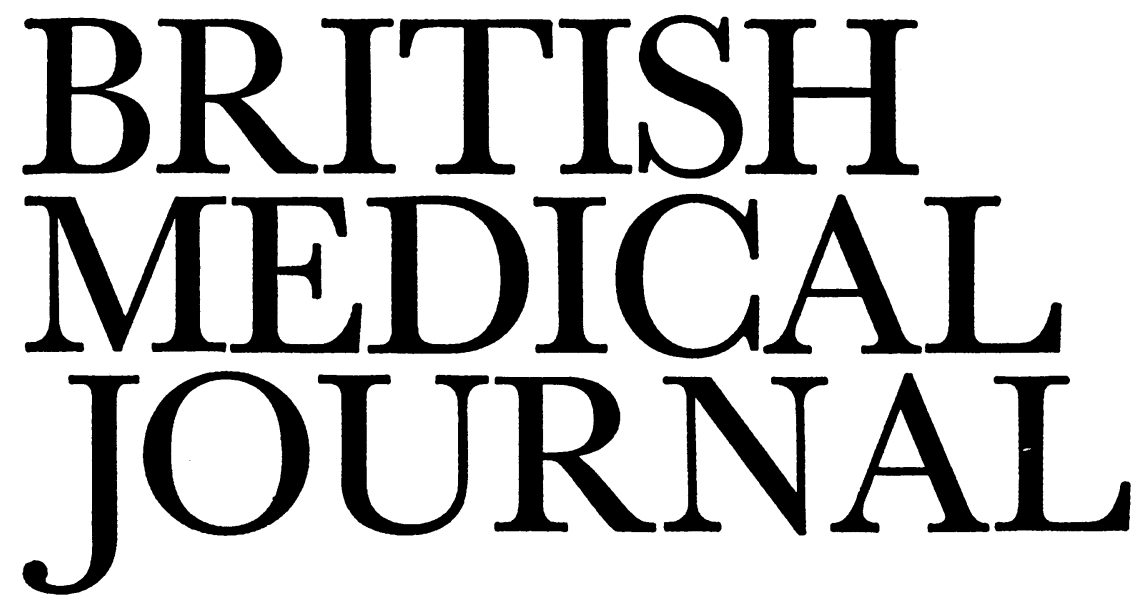

\title{
Detection of Hypertension in Childhood
}

There is already some evidence that treating mild hypertension in young adults may help to reduce complications in later life, so that perhaps treatment in childhood might be even more effective. This is a long way off as a practical therapeutic possibility, and the groundwork could be laid for it only if it were true that a relatively high blood pressure in childhood predisposed to a relatively high blood pressure in early adult life. The possibility that it might do so follows from observations that blood pressure in early adult life is statistically correlated (though only very roughly) with that observed in the fourth decade and after. Moreover, when the blood pressure is raised in early adult life, even though it is within the normal range, it may rise more rap:dly with the passage of time. Thus, from early adult life onwards the higher the blood pressure, the faster and further it rises with the passage of time.

Recently a study from Canada by Carol Buck ${ }^{1}$ has provided suggestive evidence that, if raised blood pressure is recorded at the age of 5 , it is likely to be still above the average at the age of 11-12. However, the increased blood pressure Buck observed was not very remarkable. For those children whose blood pressure at the age of 5 was over one standard deviation above the mean for their group Buck observed that the blood pressure at age 12 was approximately $9 \mathrm{~mm}$ systolic and $6 \mathrm{~mm} \mathrm{Hg}$ diastolic above the average for the age. Though statistically it seems probable that a relatively high blood pressure at the age of 5 predisposes to higher blood pressures throughout childhood and probably into early adult life, it would be unwise in the present state of knowledge to embark on a clinical trial of the treatment of marginal hypertension in childhood with drugs.

The argument could be put forward that, if children who might later be at risk for hypertension could be identified early, simpler prophylactic measures should be taken. For instance, the amount of salt in the diet might be kept low, ard at a later stage girls could be advised not to start taking the contraceptive pill. But for any screening procedure to be worthwhile its expense, inconvenience, and perhaps also its potential for causing hypochondriasis have to be weighed aga'nst the possible benefits in life expectancy. On this score alone it is difficult to recommend wholesale investigation or screening of the blood pressure of young children.
The most that might be recommended is that it should be taken annually in children when the family history is extremely strong-for example, with both parents under treatment for hypertension. Whether it would be reasonable to recommend treatment for a 12-year-old with a blood pressure of, say, $120 / 80 \mathrm{~mm} \mathrm{Hg}$ is debatable. At present treatment would not be reasonable except for higher blood pressures. The number of children likely to be found below the age of 10 , or indeed during the second decade, in need of treatment by active drugs is likely to be exceedingly small. In view of the expense and inconvenience alone screening would be difficult to justify. It would seem that for hypertension in childhood much the same applies as for hyperlipidaemia in childhood, a subject recently discussed in these columns. ${ }^{2}$ A raised serum cholesterol early in childhood can statistically predict a raised cholesterol later in childhood; but attempts to lower the serum cholesterol by active means are scarcely justifiable.

The situation may change in the next decade, because if the treatment of hypertension in young adults comes to be accepted as beneficial and an established part of medical practice, the screening of 20-year-olds will be easily justified, for it is easy to control blood pressure elevations of a mild degree in young adults. For example, guanethidine 10 $\mathrm{mg}$ daily, or perhaps propranolol $20 \mathrm{mg}$ twice daily, or bendrofluazide $5 \mathrm{mg}$ daily will in many cases restore normal levels of blood pressure without any detectable side effects. If the blood pressure is left untreated until it is perhaps $160 / 110 \mathrm{~mm} \mathrm{Hg}$ at the age of 35 , it may no longer be easy to restore it to a normal value for the age without at least some unpleasant side-effects.

The possible long-term ill-effects of drugs in common use need to be borne in mind. At present we do not know whether, for example, starting small doses of guanethidine in childhood might interfere severely with fully adequate sexual function in later life. Nor do we know whether the use of beta-blockers over several decades has long-term illeffects, perhaps on the brain. And we do not know whether the possible risk of potassium depletion and impairment of glucose tolerance by thiazide diuretics over many decades may not in itself reduce life expectancy. The earlier the physician starts to treat hypertension, and the milder the degree of hypertension, the greater is his responsibility to 
ensure that the treatment is justifiable. At present the routine screening of blood pressure in normal children or teenagers has no prophylactic value.

1 Buck, C. W., Fournal of Chronic Diseases, 1973, 26, 101.

2 British Medical fournal, 1973, 1, 690

\section{Antibiotic-induced Meningitis}

Among the many and varied side effects of antibiotic treatment a predisposition to superinfection with bacteria resistant to the antibiotic plays a considerable part. It is a novel idea that a fresh infection arising during treatment should be caused by a sensitive organism and should involve the meninges. Apparently cephalothin is the only antibiotic against which this accusation can be levelled.

A series of five cases in which meningitis developed during treatment with cephalothin for infection elsewhere is reported by R. J. Mangi and colleagues, ${ }^{1}$ of the Yale University School of Medicine, and they cite three earlier single case reports of the same condition, the meningitis having been pneumococcal in one and meningococcal in the others. Their own patients were a man of 72 with multiple myeloma, a man of 62 suspected of cholecystitis (this diagnosis was not confirmed, and no other is mentioned), a 56-year-old male diabetic and alcoholic, a woman of 20 with Hodgkin's disease receiving multiple chemotherapy, and a 49-year-old female diabetic and alcoholic with hepatic cirrhosis. The diagnosis in each except the second was pneumonia, and not only sputum but blood cultures were positive in all four, the organism found being a pneumococcus in two, a Klebsiella, and in the patient with Hodgkin's disease Listeria monocytogenes. These were the organisms subsequently found in the cerebrospinal fluid; that from the patient suspected of cholecystitis was a meningococcus. All were treated with intravenous cephalothin, usually in a dose totalling $6 \mathrm{~g}$ daily, one patient receiving kanamycin and one gentamicin in addition. There were no signs of disease of the central nervous system when treatment began, and in three patients lumbar puncture had yielded a normal fluid. The intervals between the start of trearment and the first signs of meningitis were 24, 40, and 42 hours and four and five days. All the patients except the last-named recovered after treatment with ampicillin (given to three, in one combined with gentamicin) or chloramphenicol.

The organisms isolated in all five cases are said to have been sensitive to cephalothin, but these tests were performed only by the Kirby-Bauer method, which employs highcontent discs. It would be more helpful to know what were the minimum inhibitory concentrations determined by an accurate dilution method. Likewise, there is no information about the concentrations attained by cephalothin in the cerebrospinal fluid during treatment. According to the authors "there seems to be wide variation in the degree of penetration of cephalothin across the blood-brain barrier," but a fair idea of what is to be expected can be got from studies of experimental meningitis. S. Oppenheimer and colleagues $^{2}$ found that in dogs with pneumococcal meningitis the levels attained by cephaloridine, cephalothin, and methi- cillin were respectively $10.9,5.6$, and $2.9 \%$ of those in the blood. Thus cephalothin as an agent for treating meningitis appears to be inferior to its near relative cephaloridine. The levels attained by any penicillin or cephalosporin in the absence of meningitis are well known to be much lower. Thus when the meninges were initially invaded the concentration present may well have been subinhibitory. A believer in the applicability of the Arndt-Schultz law to antibiotics, kleine Dosen reizen grosse Dasen lähmen ("small doses stimulate, large doses damage"), at least in some situations, might reasonably suspect that this low concentration had an actually stimulating effect on the bacteria. ${ }^{3}$ Something seems to be missing in the argument about the causation of this condition. Could this be it?

The paper concludes with a discussion of the place of cephalothin in the treatment of meningitis, which is admitted to be limited. Some might say that it has none. But what is in question here is not the treatment of meningitis but that of infections located elsewhere which may apparently be followed by meningitis if cephalothin is used in their treatment. It seems to have become fashionable to treat a variety of acute infections with cephalothin, often together with an aminoglycoside, as in two of these cases. Admittedly this is a combination with a very wide spectrum, but is it good routine practice? By a strange coincidence two reports from France, coming from Paris ${ }^{4}$ and Rouen 5 have appeared simultaneously in British journals, each describing three patients in whom treatment with cephalothin and gentamicin caused acute renal failure. The diagnoses in these six cases were staphylococcal septicaemia in two, pneumonia, peritonitis, enteritis, and ulcerative colitis. It seems thus that in France this combination is highly regarded for a variety of indications. Admittedly it is a good one for a life-endangering infection which could be, for instance, either staphylococcal or coliform, until the aetiology is known, and might then still be indicated for its synergic effect on a staphylococcus. Many other bacteriological diagnoses would call for a change of treatment as soon as they are made. If, for instance, the cause proves to be a pneumococcus, where is the need for anything but penicillin? For some coliform infections, and particularly Pseudomonas, gentamicin could be continued, but with carbenicillin replacing cephalothin. There would be few cases in which treatment with cephalothin and gentamicin should be continued without confirmatory evidence of the necessity for it.

${ }^{1}$ Mangi, R. J., Kundargi, R. S., Quintiliani, R., and Andriole, V. T., Annals of Internal Medicine, 1973, 78, 347

2 Oppenheimer, S., Beaty, H. N., and Petersdorf, R. G., Journal of Laboratory and Clinical Medicine, 1969, 73, 535 .

3 Garrod, L. P., British Medical fournal, 1951, 1, 205.

4 Kleinknecht, D., Ganeval, D., and Droz, D., Lancet, 1973, 1, 1129.

5 Fillastre, J. P., et al., British Medical fournal, 1973, 2, 396.

\section{Links Overseas}

Just 110 years ago the B.M.A. founded its first overseas Branch, in Bengal. Though this failed to survive a local quarrel over a paper on homoeopathy a few years later, other branches followed throughout the British Empire and subsequent Commonwealth. The next to be founded, the Jamaica Branch in 1877, continues today as the Medical Association of Jamaica, affiliated to the B.M.A. To such good effect did the 